Research Articles: Behavioral/Cognitive

\title{
Increased functional connectivity of the intraparietal sulcus underlies the attenuation of numerosity estimations for self-generated words
}

https://doi.org/10.1523/JNEUROSCI.3164-20.2021

Cite as: J. Neurosci 2021; 10.1523/JNEUROSCI.3164-20.2021

Received: 18 December 2020

Revised: 29 June 2021

Accepted: 1 July 2021

This Early Release article has been peer-reviewed and accepted, but has not been through the composition and copyediting processes. The final version may differ slightly in style or formatting and will contain links to any extended data.

Alerts: Sign up at www.jneurosci.org/alerts to receive customized email alerts when the fully formatted version of this article is published. 


\title{
Increased functional connectivity of the intraparietal sulcus underlies the
}

\author{
attenuation of numerosity estimations for self-generated words
}

Abbreviated title: Attenuation of numerosity estimations

\section{Authors}

Giedre Stripeikyte $^{1,2}$, Michael Pereira ${ }^{1,2,3}$, Giulio Rognini ${ }^{1,2}$, Jevita Potheegadoo ${ }^{1,2}$, Olaf Blanke $^{1,2,4 *}$, Nathan Faivre ${ }^{1,2,3 *}$

\section{Affiliations}

1. Center for Neuroprosthetics, Swiss Federal Institute of Technology (EPFL), CH-1202 Geneva, Switzerland

2. Brain Mind Institute, Faculty of Life Sciences, Swiss Federal Institute of Technology (EPFL), CH-1015 Lausanne, Switzerland

3. Univ. Grenoble Alpes, Univ. Savoie Mont Blanc, CNRS, LPNC, 38000 Grenoble, France

4. Department of Neurology, University of Geneva, $\mathrm{CH}-1211$ Geneva, Switzerland

* These authors contributed equally to this study

\section{Corresponding authors}

Olaf Blanke

Bertarelli Chair in Cognitive Neuroprosthetics

Center for Neuroprosthetics \& Brain Mind Institute

School of Life Sciences

Campus Biotech

Swiss Federal Institute of Technology

Ecole Polytechnique Fédérale de Lausanne (EPFL)

$\mathrm{CH}-1202$ Geneva

E-mail: olaf.blanke@epfl.ch

\section{Nathan Faivre}

Laboratoire de Psychologie et Neurocognition

CNRS UMR 5105, UGA BSHM

1251 Avenue Centrale, 38058 Grenoble Cedex 9, France

E-mail: nathan.faivre@univ-grenoble-alpes.fr

Number of pages: 43

Number of figures: 7;

tables: 4

Number of words abstract: 194; introduction: 647; discussion: 1498

\section{Conflict of interest statement}

The authors declare no competing interests. 


\begin{abstract}
42 ABSTRACT
43 Previous studies have shown that self-generated stimuli in auditory, visual, and

44 somatosensory domains are attenuated, producing decreased behavioral and neural responses compared to the same stimuli that are externally generated. Yet, whether such attenuation also occurs for higher-level cognitive functions beyond sensorimotor processing remains unknown. In this study, we assessed whether cognitive functions such as numerosity estimations are subject to attenuation in 56 healthy participants (32 women). We designed a task allowing the controlled comparison of numerosity estimations for self

50 (active condition) and externally (passive condition) generated words. Our behavioral results

51 showed a larger underestimation of self- compared to externally-generated words,

52 suggesting that numerosity estimations for self-generated words are attenuated. Moreover,

53 the linear relationship between the reported and actual number of words was stronger for

54 self-generated words, although the ability to track errors about numerosity estimations was

55 similar across conditions. Neuroimaging results revealed that numerosity underestimation involved increased functional connectivity between the right intraparietal sulcus and an extended network (bilateral supplementary motor area, left inferior parietal lobule and left superior temporal gyrus) when estimating the number of self vs. externally generated words. We interpret our results in light of two models of attenuation and discuss their perceptual versus cognitive origins.
\end{abstract}

61 
63 We perceive sensory events as less intense when they are self-generated compared to

64 externally-generated ones. This phenomenon, called attenuation enables us to distinguish

65 sensory events from self and external origins. Here, we designed a novel fMRI paradigm to

66 assess whether cognitive processes such as numerosity estimations are also subject to

67 attenuation. When asking participants to estimate the number of words they had generated

68 or passively heard, we found bigger underestimation in the former case, providing

69 behavioral evidence of attenuation. Attenuation was associated with increased functional

70 connectivity of the intraparietal sulcus, a region involved in numerosity processing.

71 Together, our results indicate that attenuation of self-generated stimuli is not limited to

72 sensory consequences but also impact cognitive processes such as numerosity estimations. 
75 The ability to distinguish self- versus externally-generated stimuli is crucial for selfrepresentation (Kircher and David, 2003; Legrand, 2006). A typical mechanism by which stimuli generated by oneself and those caused by external sources are distinguished is sensory attenuation, whereby self-generated stimuli are perceived as less intense. Indeed, previous studies have shown that self-produced stimuli in the auditory (Baess et al., 2011;

Timm et al., 2014), visual (Hughes and Waszak, 2011; Benazet et al., 2016) and somatosensory domains ( Shergill et al., 2013; Kilteni and Ehrsson, 2020), are perceived as less intense compared to the same stimuli when they are externally generated. Such attenuation was shown at the behavioral level and at the neural level in sensory cortical regions (e.g., auditory cortex (Rummell et al., 2016; Whitford, 2019)) as well as the thalamus, cerebellum, supplementary motor area and inferior parietal cortex (Hickok, 2012; Lima et al., 2016; Bansal et al., 2018; Brooks and Cullen, 2019).

Previous studies have shown that attenuation not only applies to stimuli that are generated by overt actions, but also extends to covert processes such as motor imagery (Kilteni et al., 2018) or inner speech (Scott et al., 2013; Whitford et al., 2017). In the latter case, attenuation of the electrophysiological (Whitford et al., 2017; Jack et al., 2019) and behavioral (Scott et al., 2013) responses corresponding to the test stimulus (phoneme) was observed when it matched the imagined cued phoneme. These studies used phonemes that are integrated into the early stages of hierarchical speech processing implying primary sensory cortices (Liebenthal et al., 2005; DeWitt and Rauschecker, 2012), where attenuation effects have been demonstrated (Rummell et al., 2016). An outstanding question is whether 
attenuation of self-generated stimuli is limited to sensory consequences (e.g., weakened percept) or whether it can also impact cognitive processes that are non-perceptual in nature such as abstract judgments or performance monitoring.

To answer this question, we investigated the cognitive function of numerosity estimations, defined as approximate judgments when counting is not involved (Dehaene, 1997).

Properties of numerosity estimations such as innateness, amodality, or precision that linearly decreases with increasing numerosity have been described (Anobile et al., 2016; Burr et al., 2018). Extensive neuroimaging work has also established that brain areas in the intraparietal sulcus (IPS) region play a key role in numerosity processing (for review, see Arsalidou \& Taylor, 2011). If attenuation for self-related functions extends to higher-level cognitive processes such as numerosity estimations, we would expect the number of items that are self-generated to be underestimated compared to externally-generated items in relation to modulation of IPS activity. Thus, this study aimed at investigating whether numerosity estimations of self-generated words were attenuated compared to numerosity estimations of passively heard words, thereby demonstrating that self-attenuation applies to cognitive processes beyond sensory processing.

To investigate which brain regions and networks showed activity related to attenuation processes, we designed a functional magnetic resonance imaging (fMRI) paradigm allowing the controlled comparison of numerosity estimations of either self-generated words (active condition) during a phonetic verbal fluency task or externally-generated words (passive condition) while passively listening to a stream of words. Additionally, we asked participants to evaluate the error they could have made in their estimation (performance monitoring). Assuming that attenuation occurs during numerosity estimations (active condition), we 
predicted that participants would underestimate the number of self- vs. externally-

121

122 generated words and explored possible effects on performance monitoring. As performance monitoring is better for self-initiated vs. observed processes (Pereira et al., 2020), we expected to find a stronger relationship between the reported and actual number of words in the active vs. passive condition. At the neural level, considering that sensory attenuation of self-generated stimuli involves the corresponding sensory brain regions (e.g., primary auditory cortex for attenuated sounds, see Rummell et al., 2016; Whitford, 2019), we expected to find reduced BOLD signal during the active vs. passive condition in areas responsible for numerosity processing including the IPS. 


\section{METHODS}

\section{Participants}

Three independent participant groups were tested in this study. First, we performed a behavioral pilot experiment in a mock scanner, where we tested 17 (6 women) participants (age range: $18-28$ years; $M=23$ years, $\mathrm{Cl}(95 \%)=[23,24]$ ); schooling level varied between 13 and 21 years; $M=17$ years, $\mathrm{Cl}(95 \%)=[16,18])$. During the main $\mathrm{fMRI}$ experiment, we studied 25 (14 women) participants (age range: $18-37$ years; $\mathrm{M}=23$ years, $\mathrm{Cl}(95 \%)=[22$, 26]; schooling level varied from 12 to 22 years: $M=17$ years, $\mathrm{Cl}(95 \%)=[16,18])$. $A n$ additional control experiment in the mock scanner was performed, where 14 (12 women) participants were tested (age range: $18-29$ years; $\mathrm{M}=24$ years, $\mathrm{Cl}(95 \%)=[22,26]$ ); schooling level varied between 10 and 23 years; $M=16$ years, $\mathrm{Cl}(95 \%)=[14,18])$. All participants were right-handed according to the Edinburgh Hand Preference Inventory (Oldfield, 1971) and native French-speaking healthy volunteers with no history of neurological or psychiatric disease and no recent reported history of drug use. Participants had normal or corrected-to-normal vision and no claustrophobia. All participants were naive to the purpose of the study, gave informed consent in accordance with institutional guidelines and the Declaration of Helsinki, and received monetary compensation (20 CHF / hour). The study was approved by the local ethical committee of the canton Geneva (protocol ID: 2015-00092).

\section{Experimental task}

The experiment was performed in a mock scanner environment (both for the behavioral pilot experiment and the training for the main $\mathrm{fMRI}$ experiment) and in the MRI scanner 
(main fMRI experiment). The task consisted in phonetic verbal fluency and passive word listening parts, followed by numerosity and error estimations (Figure 1). The task comprised two conditions (20 trials each) during which participants either covertly generated words (active condition) or listened to pre-recorded words (passive condition). Each trial started with a randomly jittered inter-trial interval varying between $4 \mathrm{~s}$ and $4.75 \mathrm{~s}$, followed by an audio cue $(2 \mathrm{~s})$ indicating which of the active or passive condition will follow, and a recorded cue letter ( 2 s). Next, the word generation phase lasted between $20 \mathrm{~s}$ and $35 \mathrm{~s}$; in the active condition, participants had to covertly generate words starting with the cued letter (Figure 1, top). For each word they covertly generated, participants were asked to press a response button, which gave us access to the actual number of generated words. In the passive condition, a series of pre-recorded audio words were played to the participants, who were asked to press the response button for every word that contained the cue letter (Figure 1, bottom). We avoided asking participants to detect words starting with the cued letter, as this could have influenced word generation in the next active trials with the same cued letter. In both conditions, the end of the generation/listening phase was indicated by an audio cue $(0.5 \mathrm{~s})$. After that, participants first reported the estimation of a total number of words generated (active condition) or heard (passive condition). For this, they used two buttons that moved a slider displayed on the screen. The slider was presented as a random integer (ranging from 0 to 20), which could be changed in value by pressing the response box buttons (left button - decrease the value, right button - increase the value). This was followed by an error estimation, where participants were asked to evaluate their performance on the numerosity estimation by estimating the magnitude of the error they thought they might have made (in number of words). For this, an automatically sliding bar was presented, and the participant had to select with one button press the desired value 
(e.g., +/- 2 words error). Values varied from +/- 0 words (e.g., numerosity response judged as correct) to +/- 5 words mistaken. The total time for numerosity and error estimations were restricted to a maximum of $7 \mathrm{~s}$. Except for the numerosity and error estimation periods, participants were asked to perform the task with eyes closed.

\section{Stimuli}

All stimuli were prepared and presented using Matlab 2016b (mathworks.com) and the Psychtoolbox-3 toolbox (psychtoolbox.org; Brainard 1997; Kleiner et al. 2007; Pelli 1997). Twenty different cue letters were used for active and passive conditions. The same cue letter was used once during the active and once during the passive condition in the counterbalanced order. Played back words during the passive condition were chosen from a list of 420 French words (Ferrand and Alario, 1998). The audio stimuli presented during the task were recorded by male and female native French speakers in a neutral manner and registered in wav format with $11025 \mathrm{~Hz}$ sampling frequency. The gender of the voice pronouncing words in the passive condition was matched to the participants' gender. During the experiment, participants were equipped with MRI compatible earphones and report buttons for the right hand.

\section{Procedure}

Participants were trained in a mock scanner prior to the main fMRI experiment in order to familiarize themselves with the task. They were asked to perform four trials of the task during the training (twice for each condition), with the cue letters ' $j$ ' and ' $k$ '. These letters were not used later during the main $\mathrm{fMRI}$ experiment. We note that participants were 
instructed by the experimenter not to count nor use any strategy to try to remember the number of words generated/heard while performing numerosity and error estimations.

The main experiment consisted of three runs lasting approximately $15 \mathrm{~min}$, each with short breaks in-between. The total duration of a trial varied between 40 and $55 \mathrm{~s}$ due to pseudorandomized time for the word generation/ listening phase. This time variability was introduced to avoid habituation and predictability for the number of generated/heard words and to decorrelate hemodynamic activity related to word generation/listening and numerosity estimations. The experiment was designed in 10 blocks with 4 trials of the same condition per block. The blocks of the active condition trials always preceded the blocks of the passive condition, allowing us to use the number and pace of generated words that were recorded based on participant's button presses to playback the words during the next block of the passive condition. The order of the number of words played with their corresponding pace during the passive condition was shuffled within the block. This was done to ensure that participants could not recognize whether the number and pace of the played words matched the preceding active condition block. Additionally, to avoid repetition effects between blocks, the cue letters between two consecutive blocks were different, meaning that the cue letters in a given active block were not used in the following passive block.

After the main experiment, a standard phonemic verbal fluency (generation time of $60 \mathrm{~s}$, cue letter ' $p$ ') test (Lezak, 1995) was performed overtly to verify that subjects understood the task correctly. Overall, the experiment lasted approximately $1 \mathrm{~h} 30 \mathrm{~min}$ (MRI session) and $1 \mathrm{~h}$ in the mock scanner (pilot session). The pilot mock scanner study contained the 
same procedure as the main MRI experiment, except for the shorter breaks between the runs since there was no scanning involved.

The sample size for the main $\mathrm{fMRI}$ experiment was based on a recent study from our group in which similar conditions were contrasted (e.g., judgement of self-generated vs. observed decisions; Pereira et al., 2020).

\section{Control order experiment}

An additional control experiment in the mock scanner was performed to rule out the possibility that repetition effects occurred between consecutive blocks. It consisted only of passive condition trials, which were divided into 'random' and 'repeated' blocks. The 'random' block trials were designed by pseudo-randomly generating the number of words played (mean of 10 words/block ranging from 6 to 20 words per trial) and their playing pace (total time of playing varied between $20 \mathrm{~s}$ and $35 \mathrm{~s}$ ) to match the main fMRI experiment. 'Repeated' blocks consequently followed 'random' blocks, with the same number of words and word playing pace as in the preceding random block but with a shuffled order. This control experiment consisted of 2 'random' and 2 'repeated' blocks, with 4 trials/block, and lasted approximately $30 \mathrm{~min}$.

\section{Behavioral performance measures}

Most statistical frameworks to analyze performance monitoring have been developed for discrimination tasks with a binary response (e.g., Fleming and Lau, 2014 for a review). In the following, we propose two indices of numerosity performance and performance monitoring to analyze ordinal data (e.g., number of words). In addition to the prerequisites described 
below, these indices were defined at the single-trial level so they could serve as parametric regressors of interest in the $\mathrm{fMRI}$ analysis.

The numerosity performance index is a normalised accuracy ratio reflecting how correctly participants estimated the number of words during the generation/listening phase. For each trial, we wanted signed numerosity performance to be proportional to the difference between the reported number of generated/heard words (numerosity estimation; $[\mathrm{N}]$ ) and the actual number of words generated/heard [W]. We normalized this difference by the sum of numerosity estimation and actually generated/heard number of words [N+W] (equation 1) to give more weight to errors made about low numbers of words (e.g., an error of $+/-2$ given a numerosity estimation of 8 has higher magnitude than an error of $+/-2$ given a numerosity estimation of 16; Figure 1-1 A). This normalization allowed us to assess attenuation effects independent of the number of generated words, given that the precision of numerosity estimation linearly decreases with increasing number of items (e.g., Piazza et al., 2004). Negative numerosity performance values thus reflected an underestimation of generated/heard words, and positive values reflected an overestimation of generated/heard words. In contrast, null numerosity performance values reflected correct answers about the number of generated/heard words.

numerosity performance $=\frac{N-W}{N+W}$ (equation 1)

Additionally to the numerosity performance index, we derived separate measures of accuracy $[N-W]$ and accuracy ratio $[(N-W) / W]$ to assess the generalizability of our findings. 
Performance monitoring reflected how well participants estimated an error about their previous performance. We defined it as the absolute value of the difference between the error estimation $[E]$ and (accuracy $[N-W]$ ), normalized by the sum of the numerosity estimation and words generated/heard $[\mathrm{N}+\mathrm{W}]$ ) (equation 2). Normalization was done in order to consider the difficulty; the same error made when estimating the low number of words or high number of words should be penalized proportionally. A performance monitoring value of 0 reflected ideal error tracking, whereby participants correctly estimated the error made during the numerosity estimation. An increase in performance monitoring value represented an increase in error magnitude while estimating the difference between numerosity estimation and the actual number of words generated/heard (Figure 1-1 B).

performance monitoring $=\left|\frac{E-|N-W|}{N+W}\right|$ (equation 2)

Data cleaning was performed prior to statistical analysis: trials for which participants did not generate at least five words or failed to answer numerosity or error estimations within the time limit were excluded from behavioral and fMRI analysis (in total, $2.44 \pm 1.87$ trials/subject were excluded). The threshold of 5 words was selected according to the working memory capacity of $5 \pm 2$ items (Cowan, 2010). Considering all participants' data, $6.1 \%$ of all trials were discarded.

\section{Behavioral data analysis}

All continuous variables (numerosity performance, accuracy, accuracy ratio and performance monitoring) were analyzed using linear mixed-effects regressions with 
condition ("active", "passive" or "random", "repeat" for the control order experiment) as a fixed effect and a random intercept by participant and condition. The inclusion of additional random effects was guided by model comparison and selection based on Bayesian Information Criteria. Analyzes were performed using the Ime4 (Bates et al., 2015) and ImerTest (Kuznetsova et al., 2017) packages in R (R-project.org). The significance of fixed effects was estimated using Satterthwaite's approximation for degrees of freedom of F statistics (Luke, 2017).

Bayesian analysis was performed using the brms package (Bürkner, 2017) in R to examine the occurrence of possible repetition effects between random and repeated passive blocks in the control experiment. Namely, we conducted a Bayesian linear model of numerosity performance with condition ("random", "repeated") as fixed effects and a random intercept by participant and condition with four chains of 10000 iterations including 2000 warmup samples. We made a prior assumption that underestimation would be observed in the passive condition (prior with Gaussian distribution of mean $=-0.032$ and SD $=0.156$ ), based on the difference between numerosity performance during the active and passive conditions observed in the main $\mathrm{fMRI}$ experiment.

\section{Data simulation of absolute accuracy during numerosity estimations}

To examine weather absolute accuracy (correct numerosity estimations of words generated/heard) of numerosity estimations observed in the experimental data may be approximated by a noisy sampling process rather than counting, we performed data simulations. More specifically, we simulated 1000 numerosity estimations (as the main experiment contained 40 trials/subject $* 25$ subjects) from a normal distribution centred on 10 and standard deviation (SD) varying from 0.5 to 6 with 0.1 steps. We rounded each item 
to the nearest integer and counted how many times the number 10 was obtained. The simulation was iterated 10000 times.

\section{fMRI data acquisition}

MRI data were acquired using a Siemens Magnetom Prisma 3 T scanner with a 64-channel head coil. T1 weighted (1 mm isotropic) scans were acquired using a Magnetization Prepared Rapid Acquisition Gradient Echo (MPRAGE) sequence (TR = 2300 ms; TI = 900 ms; TE $=2.25 \mathrm{~ms} ;$ flip-angle $=8$ degrees; GRAPPA = 2; FOV = $256 \times 256 \mathrm{~mm} ; 208$ slices). Functional scans were obtained using echo-planar $(\mathrm{EPI})$ sequence (multiband acceleration = 6; TR = 1000 ms; TE = 32 ms; flip-angle = 58 degrees; FOV = $224 \times 224$ mm; matrix = $64 \times 64$; slice thickness $=2 \mathrm{~mm}$; number of slices $=66$ ). The number of functional image volumes varied according to the experiment duration (2278 \pm 61 volumes).

\section{fMRI data preprocessing}

Anatomical and functional images were processed and analyzed using SPM-12 (Welcome Centre for Human Neuroimaging, London, UK). Pre-processing steps included slice time correction, field-map distortion correction, realignment and unwarping to spatially correct for head motions and distortions, co-registration of structural and functional images, normalization of all images to common Montreal Neurological Institute (MNI) space, and spatial smoothing with a Gaussian kernel with a full-width at half maximum (FWHM) of 4 $\mathrm{mm}$. Quality assurance of all EPI images was performed with the criteria of maximum $2 \mathrm{~mm}$ translation and $2^{\circ}$ rotation between volumes. In addition, an excessive movement was estimated with the mean framewise displacement (FD) (Power et al., 2012) with the exclusion threshold of $0.5 \mathrm{~mm}$. None of the subjects had a higher mean FD $(0.2 \pm 0.06 \mathrm{~mm})$ than the set threshold. 


\section{fMRI data analysis}

We used a two-level random-effects analysis. In the first-level analysis, condition-specific effects were estimated according to a general linear model (GLM) fitted for each subject. An average mask of grey matter from all subjects was built using FSL (fsl.fmrib.ox.ac.uk/fsl) and used to mask out white matter and non-brain tissues. The GLM was built using six boxcar regressors corresponding to the duration of the word generation/listening phase, numerosity estimation and error estimation in the active and passive conditions. Parametric modulators of numerosity performance and performance monitoring were included in the numerosity estimation and error estimation regressors, respectively. Further, we added regressors of no interest corresponding to audio instructions, button presses and excluded trials, plus six regressors for head motion (translation and rotation).

At the second-level (group level), we performed a one-way analysis of variance (ANOVA) with F-tests to assess main effects common to active and passive conditions and t-tests to analyze the difference between conditions (active vs. passive) for each regressor of interest: numerosity estimation and error estimation. We used a voxel-level statistical threshold of $p<0.001$ and corrected for multiple comparisons at the cluster level using family-wise error (FWE) correction with the threshold of $p<0.05$. We used the anatomical automatic labelling (AAL) atlas for brain parcellation (Tzourio-Mazoyer et al., 2002).

\section{Functional connectivity analysis}

Psychophysiological Interaction (PPI) analysis was employed to identify modulations of functional coupling between a seed region and other brain regions by experimental conditions (active vs. passive) (Friston et al., 1997). To perform this analysis we used 
generalized psychophysiological interaction (gPPI) toolbox version 13.1 (McLaren et al., 2012). Spheres of $6 \mathrm{~mm}$ radius were formed around the peak coordinates of the right IPS ( $\mathrm{x}$ $=29 ; y=-65 ; z=50)$ and the left IPS $(x=-27 ; y=-66 ; z=47)$ clusters that were identified in the second-level analyses. First-level (individual) GLM analyses were performed, including task regressors of numerosity estimation or error estimation (psychological term) and time course of the seed region (physiological term). As for other aforementioned fMRI analyses, we performed t-tests to compare the differences between conditions.

\section{Data and code availability}

The MRI data that support the findings of this study is available on a public repository on zenodo.org (https://doi.org/10.5281/zenodo.4925909). Behavioral data, analysis and task codes are available here: https://gitlab.epfl.ch/Inco-public/cognitive-attenuation.

\section{RESULTS}

\section{Behavioral results}

By design, the number of generated and heard words was matched between conditions (active: $\mathrm{M}=10.7, \mathrm{SD}=3.6, \mathrm{Cl}(95 \%)=[10.4,11.1] ;$ passive: $\mathrm{M}=10.7, \mathrm{SD}=3.5, \mathrm{Cl}(95 \%)=$ $\left.[10.4,11.0], F_{(1,884)}=0.005, p=0.94\right)$. The duration of word generation and listening did not differ between conditions either (active: $M=27.4, S D=5.6, C l(95 \%)=[26.9,27.9]$; passive: $\left.M=28.4, S D=7.0, C I(95 \%)=[27.7,29.0], F_{(1,26)}=2.04, p=0.16\right)$. Having shown that conditions were similar in terms of difficulty, we turned to the analysis of numerosity performance and performance monitoring.

Numerosity performance indices revealed that globally, participants underestimated the number of words $(\mathrm{M}=-0.05, \mathrm{SD}=0.11, \mathrm{Cl}(95 \%)=[-0.06,-0.05])$. This underestimation was 
significantly larger $\left(F_{(1,24)}=5.85, p=0.023,18\right.$ subjects out of 25 showed the effect $)$ in the active condition $(\mathrm{M}=-0.07, \mathrm{SD}=0.11, \mathrm{Cl}(95 \%)=[-0.08,-0.06])$ compared to the passive condition $(\mathrm{M}=-0.04, \mathrm{SD}=0.12, \mathrm{Cl}(95 \%)=[-0.05,-0.03])$ (Figure $2 \mathrm{~A})$. Comparable results were obtained during the pilot experiment in the mock scanner in an independent group of subjects (active: $\mathrm{M}=-0.05, \mathrm{SD}=0.01, \mathrm{Cl}(95 \%)=[-0.06,-0.03]$ ); passive: $\mathrm{M}=-0.03, \mathrm{SD}=0.10$, $\left.\mathrm{Cl}(95 \%)=[-0.04,-0.02] ; \mathrm{F}_{(1,16)}=5.80 \mathrm{p}=0.016\right)$. These two experiments suggest that numerosity estimations for self-generated words are attenuated.

We further show that the larger underestimation in the active compared to the passive condition was also found when considering the measures of accuracy (active: $M=-1.48, S D$ $=2.38, \mathrm{Cl}(95 \%)=[-1.69,-1.26]) ;$ passive: $\mathrm{M}=-0.94, \mathrm{SD}=2.59, \mathrm{Cl}(95 \%)=[-1.17,-0.71]$ $F(1,24)=4.43 p=0.045$ ) and accuracy ratio as dependent variables (active: $M=0.89, S D=$ $0.20, \mathrm{Cl}(95 \%)=[0.87,0.91]) ;$ passive: $\mathrm{M}=0.95, \mathrm{SD}=0.23, \mathrm{Cl}(95 \%)=[0.93,0.97] ; \mathrm{F}(1,24)=$ $7.44 p=0.011)$

We then assessed the linear relationship between the reported (numerosity estimation) and actual number of words (Figure 2 B). Besides a main effect of words $\left(F_{(1,908)}=1042, p<\right.$ $0.001)$ and of condition $\left(F_{(1,198)}=8.25, p=0.0045\right)$, we found an interaction between condition and generated/heard words $\left(F_{(1,802)}=4.3, p=0.038\right)$. This interaction was driven by a steeper slope in the active condition $(\mathrm{M}=0.62, \mathrm{Cl}(95 \%)=[0.57,0.67])$ compared to the passive condition $(\mathrm{M}=0.55, \mathrm{Cl}(95 \%)=[0.50,0.60])$ (Figure $2 \mathrm{C})$, which indicates better numerosity tracking for self-generated words during the main fMRI experiment (a slope of value 1 reflecting ideal performance). We note that this interaction did not reach significance in our pilot experiment $\left(F_{(1,350)}=1.10, p=0.66\right)$, which calls for interpreting this result with caution. 
Next, we investigated whether performance monitoring varied between conditions, by quantifying how well participants were able to track the error made when estimating the number of generated/heard words. We found no differences in performance monitoring $\left(F_{(1,24)}=1.09, p=0.3\right)$ between the active $(M=0.07, S D=0.06, C l(95 \%)=[0.07,0.08])$ and passive $(\mathrm{M}=0.08, \mathrm{SD}=0.06, \mathrm{Cl}(95 \%)=[0.07,0.09])$ conditions during the main $\mathrm{fMRI}$ experiment, nor during the mock scanner pilot experiment $\left(F_{(1,16)}=0.78, p=0.39\right.$; active: $M$ $=0.07, \mathrm{SD}=0.06, \mathrm{Cl}(95 \%)=[0.06,0.08]$; passive: $\mathrm{M}=0.06, \mathrm{SD}=0.06, \mathrm{Cl}(95 \%)=[0.06,0.07])$. This confirms the absence of evidence supporting an effect of attenuation on performance monitoring.

Finally, we carried out a control task for word generation outside the scanner, during which participants had to perform a standard verbal fluency task overtly with the cue letter ' $\mathrm{p}$ '. Comparing the number of words generated, starting with the letter ' $p$ ' overtly (outside the scanner) and covertly (during the scanning), we did not observe any significant differences between the number of words generated overtly $(\mathrm{M}=12.6, \mathrm{SD}=3.79, \mathrm{Cl}(95 \%)=[10.9$, 14.1]) and covertly $\left(M=12.3, S D=3.96, C l(95 \%)=[10.6,13.9]\right.$; paired t-test; $t_{(24)}=-0.4$; $p=0.69)$. This control task confirms that subjects did generate words covertly and comparably to overt fluency, as instructed.

Control order experiment. The global underestimation $(\mathrm{M}=-0.06, \mathrm{SD}=0.11, \mathrm{Cl}(95 \%)=[-$ $0.08,-0.05])$ was replicated as in the main experiment. This underestimation was not different $(F(1,117)=0.03, p=0.87)$ between 'random' $(M=-0.06, S D=0.12, C l(95 \%)=[-$ $0.09,-0.04])$ and 'repeated' $(\mathrm{M}=-0.07, \mathrm{SD}=0.12, \mathrm{Cl}(95 \%)=[-0.09,-0.04])$ blocks, with a Bayes factor BF10 $=0.09$ supporting the absence of difference between conditions. 
Data simulation of absolute accuracy during numerosity estimations. As can be seen on the

Figure 3 , the value of 10 occurred in $19.5 \%$ of all data points when drawn from a normal distribution with $\mathrm{SD}=2$, similarly to the observed empirical data.

\section{fMRI results}

\section{Numerosity performance}

Brain activity during the numerosity estimation phase was widespread, irrespective of the experimental condition (Table 1-1). Differences between conditions revealed widespread relative deactivations in the active compared to the passive condition in the bilateral parietal cortex including IPS, middle-superior temporal gyri, precuneus, cerebellum, middle cingulate gyri, SMA, insula, middle-superior frontal gyri, hippocampus, caudate nucleus, putamen (for the detailed list of all areas see Table 1).

To avoid contaminating the results with inherent differences between the active and passive conditions that are not specific to numerosity processes, we looked for brain activity parametrically modulated by numerosity performance. We found a single brain region with such a pattern of activity, namely the right IPS, whose activity was negatively correlated with numerosity performance (main effect $F=20.0, p_{F W E}=0.042, k_{v o x}=148, x=29, y=-65, z$ = 50; Figure 4 A, B). The same pattern was found bilaterally when using a less stringent threshold ( $p<0.005$ peak level uncorrected; Figure 4 C, Table 2). Unlike our prediction, IPS activation did not differ between the active and passive conditions $\left(\mathrm{p}_{\mathrm{FWE}}>0.05\right)$, suggesting that this effect was independent of whether words were heard or actively generated. 
Using PPI analysis, we investigated whether the functional connectivity of the right IPS with other brain regions differed between the active and passive conditions. This analysis revealed that bilateral supplementary motor area (SMA), left inferior parietal lobule (IPL) and left superior temporal gyrus (STG) had increased connectivity with the right IPS in the active compared to the passive condition (Figure 5, Table 3). No effect in the other direction was observed.

\section{Performance monitoring}

Besides numerosity performance, we also quantified brain activity during the error estimation phase. We found widespread cortical and subcortical activations during this phase compared to baseline, irrespective of the experimental condition (Table 4-1).

Moreover, bilateral insula and right putamen were significantly less active during the active compared to the passive condition (Figure 6, Table 4). The left caudate nucleus showed the opposite pattern, with higher activity in the passive than in the active condition.

To avoid confounding factors between the active and passive condition, we looked more specifically at parametric modulations of error monitoring. We observed that BOLD signal in the left IPS was more related to performance monitoring in the active compared to the passive condition $\left(t=4.02, \mathrm{p}_{\mathrm{FWE}}=0.041, \mathrm{k}_{\mathrm{vox}}=198, \mathrm{x}=-27, \mathrm{y}=-66, \mathrm{z}=47\right.$; Figure 7$)$. Interestingly, this activation cluster spatially overlapped with the observed activation cluster related to the main effect of numerosity performance when applying a lower statistical threshold (exploratory analysis, $\mathrm{p}<0.005$ peak level uncorrected, pFWE $<0.05$ cluster level) (see Figure 4 C). 
462 Lastly, results from the PPI analysis using the left IPS as a seed ROI did not reveal any

463 functional connectivity differences between the active and passive conditions during error

464 estimation.

465

466 


\section{DISCUSSION}

The present study examined whether attenuation of self-generated stimuli impacts cognitive processes beyond the traditionally investigated sensory processes, namely numerosity estimations and performance monitoring. To this end, we developed an experimental paradigm allowing the controlled comparison of numerosity estimations and performance monitoring regarding self- and externally-generated words while acquiring fMRI data. We found that participants more strongly underestimated the number of selfcompared to externally-generated words, providing behavioral evidence that numerosity estimations are indeed subject to attenuation. As expected, numerosity performance was associated with hemodynamic activity changes in IPS. Furthermore, a network including the bilateral SMA, left IPL and left STG showed increased functional connectivity with the right IPS during numerosity estimations for self-generated words, suggesting that numerosityrelated attenuation involves this neural network. Finally, by asking participants to monitor the accuracy of their own numerosity estimations, we found equivalent performance monitoring for self- and externally-generated words. Although no difference was found at the behavioral level, we found that performance monitoring was associated with increased IPS activity in the active vs. passive condition.

\section{Behavioral and neural markers of attenuated numerosity estimations}

Participants underestimated the number of words they generated or heard. Such

underestimations were previously described regarding the numeric estimation of perceptual quantities (e.g., number of dots or sequences of sounds) to discrete measures (e.g., Arabic numeral) (Castronovo and Seron, 2007; Reinert et al., 2019). In the present study, we found that word numerosity underestimation was stronger in the active compared to the passive 
condition, in line with our hypothesis that attenuation of self-generated stimuli may extend to higher-level cognitive functions (Kilteni et al., 2018; Jack et al., 2019). Of note, this underestimation could not be due to simple repetition effects between successive blocks of active and passive condition trials, as a control experiment showed no differences in numerosity estimations between repeated blocks of passive condition trials.

Attenuation of self-generated stimuli has been mostly investigated for sensory processes and refers to the diminished behavioral and neural responses associated with selfgenerated compared to externally-generated stimuli (Timm et al., 2014; Benazet et al., 2016). Importantly, attenuation has recently also been described for imagined actions in the absence of overt actions (e.g., Kilteni et al., 2018; Jack et al., 2019;). For example, imagined self-touch was felt as less intense compared to externally applied touch (Kilteni et al., 2018). Similarly, imagined speech elicited reduced electrophysiological signals related to auditory processing (Whitford et al., 2017). These studies, however, investigated cognitive processes intimately linked to sensorimotor systems (e.g., imagined movement and touch), yet, to the best of our knowledge, it was unknown whether comparable mechanisms of attenuation also affect cognition beyond sensorimotor processing, such as numerosity estimations with no or less obviously implicated sensorimotor processes (e.g., Dehaene, 1997). Here we show that differential attenuation can be observed for cognitive processes such as numerosity estimations that depend on whether they are self-generated or not. As of today, there is no consensus to explain how the brain attenuates expected (e.g., self-generated) stimuli while remaining sensitive to unexpected ones (for a recent review and unifying theoretical account see Press et al., 2020). Among the so-called cancellation theories, the internal 
513 forward model (Farrer and Frith, 2002; Miall and Wolpert, 1996) proposes that corollary

514 discharges related to action are used to predict the sensory consequences of that action.

515 When such predictions match the actual sensory feedback from the action, its sensory

516 consequences are attenuated, and the action is perceived as self-generated (Wolpert and

517 Flanagan, 2001). The forward model thus proposes to link sensory attenuation to the

518 sensory predictions generated by a neural comparator. An analogous mechanism has been

519 proposed to account for attenuation for covert actions such as motor imagery (Kilteni et al.,

520 2018) or inner speech (Tian and Poeppel, 2010). The present data show that attenuation

521 exists beyond overt and covert actions, raising the possibility that the forward model

522 extends to repetitive cognitive activity (e.g., fluency and related numerosity estimations).

523 One possibility, similar to what has been described for inner speech, would be that

524 numerosity underestimation stems from a weaker perceptual representation of self-

525 generated words. In other words, attenuation may not impact numerosity estimations

526 directly, but rather through a decreased representation of self-generated words, which in

527 turn may lead to attenuation of numerosity estimations.

528 A second account could be that mental operations have a gating effect (Cromwell et al.,

529 2008), independent from predictive mechanisms, thereby directly affecting the strength of

530 the mental representations of self-generated words. A similar gating mechanism has

531 recently been shown to offer a plausible alternative to forward model accounts of sensory

532 self-attenuation (Thomas et al., 2020). Finally, the general mechanisms put forward by the

533 predictive coding account of active inference to explain sensory attenuation may also apply

534 to non-perceptual cognitive attenuation such as the one we observed (Brown et al., 2013). 
At the neural level, the IPS showed activity related to numerosity performance, in line with previous studies (Cohen and Dehaene, 1996; Piazza et al., 2006). This relation, however, was not modulated by condition (active vs. passive), as one could have expected based on previous reports showing that sensory attenuation coincides with decreased activity in the corresponding sensory area (e.g., attenuation in primary auditory cortex during selfgenerated auditory stimuli; Rummell et al., 2016; Whitford, 2019). Importantly, we found differences in functional coupling during numerosity estimations of the IPS with a network of brain regions between the active and passive conditions. The increase in coupling during the active condition occurred between the IPS and a network comprising the SMA, IPL and STG, known to be involved in predictive processing, for example of self-generated auditory and imagery speech (Lima et al., 2016; Tian et al., 2016). Although previous neuroimaging studies have mainly shown attenuated brain activity for self-generated actions (e.g., Whitford, 2019), increases in functional connectivity such as we describe have been reported in the primary auditory cortex during attenuated speech (van de Ven et al., 2009), or of somatosensory cortex during attenuated touch (Kilteni and Ehrsson, 2020). Thus, increased functional connectivity in a network centred on the key numerosity region, IPS, and that has been associated with speech-related processing further supports that numerosity underestimation stems from a process related to attenuation when participants self-generate words.

Besides numerosity underestimation, we observed that participants formed more accurate numerosity judgments for self- vs. externally-generated words: while participants' word estimations were lower in the active condition than in the passive condition, the relation 
between their estimated number of words and the actual number of words was better in the active condition vs. passive condition, suggesting a sharper representation of the number of self-generated words. This result corroborates recent findings showing better monitoring for decisions that are committed rather than observed (Pereira et al., 2020). This improved monitoring of self-generated words could be related to the sharpening of expected representations known in the sensory domain (Kok et al., 2012) or to a selfgeneration effect underlying the facilitation of information encoding and enhanced recall for self-generated stimuli (Bertsch et al., 2007; Slamecka and Graf, 1978). We note that this effect was not replicated during the mock pilot experiment, which included a smaller sample of participants with a globally higher absolute accuracy. It is possible that this subtle effect could not be observed in such conditions, which warrants future investigations and cautious interpretation.

\section{Performance monitoring is modulated at the neural but not behavioral level}

Previous research has shown that both humans and non-human primates (Beran et al., 2006; Duyan and Balcı, 2019, 2018) can monitor the quality of their numerosity estimations. Thus, in addition to asking participants to estimate the number of words they generated or heard, we also asked them to estimate their own error during this process (performance monitoring). Although our behavioral results showed similar performance monitoring between conditions, we observed that performance monitoring was associated with hemodynamic activity in the left IPS predominantly in the active condition. Interestingly, this region is not typically associated with performance monitoring such as the prefrontal cortex or the insula/inferior frontal gyrus (Vaccaro and Fleming, 2018). Since activity in the left IPS is related to numerosity estimation (Cappelletti et al., 2007; Dormal et al., 2012), this 
parametric modulation could therefore represent a substrate for monitoring specific to numerosity estimations. By investigating global fMRI activity during error estimations, we also observed decreased activation in the insula and putamen and increased activity in the caudate nucleus when comparing the active vs. passive conditions. To note, the anterior insula and putamen were activated during both conditions but were attenuated in the active compared to the passive conditions. While the anterior insula activations are consistent with previous literature on performance monitoring (Ullsperger et al., 2010; Bastin et al., 2017), the findings of modulated activity in the striatum were unexpected. Both areas are known as essential for the control of goal-directed decision-making (Balleine et al., 2007; Kim and Im, 2019). Further links between performance monitoring and activity modulation between conditions in the striatum regions should be explored in future studies.

\section{Methodological considerations}

As generating vs. listening to words inherently involves different behavioral and neural processes, several aspects of our design should be considered when interpreting the current findings as reflecting attenuation per se rather than experimental confounds. At the behavioral level, the larger underestimation during the active condition was not due to difficulty differences between conditions as task difficulty was matched by design, and absolute accuracy (correct numerosity estimations of words generated/heard) was similar in both conditions. Of note, a steeper slope during numerosity estimations and number of words generated in the active condition suggests that participants better tracked their performance even when underestimating more compared to the passive condition.

Although we could not explicitly control whether participants counted the number of words they generated or heard, instructions were given not to do so. Yet, counting seems unlikely 
as absolute accuracy reached only $19.5 \%$ of all trials, which is comparable to a recent study with a similar setup (Serino et al., 2021), and much lower than what is typically observed when participants are instructed to count (e.g, Kansaku et al., 2007). Furthermore, a similar level of absolute accuracy was obtained on simulated data with numerosity estimations drawn from a normal distribution with $\mathrm{SD}=2$, suggesting that behavior in this task is approximated by a noisy sampling process rather than counting. At the neural level, to ensure that the inherent differences between conditions did not contaminate our results, we focused analyses on the numerosity and error estimation phases which were identical between conditions, instead of the word generation/listening phases. In addition, we did not base our conclusions on a direct contrast between the two conditions, but on correlations between neural activity and numerosity or error estimates.

\section{Conclusion}

Based on behavioral and neuroimaging data, we propose that higher-level cognitive functions such as numerosity estimations about the number of self-generated words are attenuated. Such attenuation involves a functional network including a key-numerosity region (IPS) and speech-related regions including the SMA, IPL and STG. While attenuating the sensory consequences of one's actions is of crucial importance for aspects of the self, such as the sense of agency, attenuating the products of one's mental activities may also be relevant to distinguish them from external sources of information. Our paradigm offers a promising tool to investigate attenuation processes related to the self in cognition and to compare and distinguish them from sensory attenuation processes. It may be of relevance to the study of clinical cases in which attenuation in sensory and cognitive domains may be 
626 is altered, including patients with psychotic symptoms like thought insertion whereby

627 thoughts are not considered as one's own, but of somebody else.

628 


\section{Acknowledgments}

The authors are grateful for the technical support during data acquisition to the Foundation Campus Biotech Geneva (FCBG) Human Neuroscience Platform of the Campus Biotech. This work was supported by the Bertarelli Foundation (grant number 532024), the Swiss National Science Foundation (grant number 3100A0-112493), National Center of Competence in Research (NCCR) "Synapsy - The Synaptic Bases of Mental Diseases" (grant number 51NF40 - 185897), and two generous donors advised by Carigest SA. Nathan Faivre has received funding from the European Research Council (ERC) under the European Union Horizon 2020 research and innovation programme (grant number 803122 ).

\section{Author Contributions}

GS, NF, GR, OB developed the study concept and contributed to the study design. Testing, data collection and data analysis were performed by GS. GS, MP, JP and NF provided methodological support. GS, NF, MP, and OB drafted the paper; all authors provided critical revisions and approved the final version of the paper for submission. 


\section{REFERENCES}

Anobile, G., Cicchini, G.M., Burr, D.C., 2016. Number As a Primary Perceptual Attribute: A Review. Perception 45, 5-31. https://doi.org/10.1177/0301006615602599

Arsalidou, M., Taylor, M.J., 2011. Is 2+2=4? Meta-analyses of brain areas needed for numbers and calculations. Neuroimage 54, 2382-2393. https://doi.org/10.1016/j.neuroimage.2010.10.009

Baess, P., Horváth, J., Jacobsen, T., Schröger, E., 2011. Selective suppression of self-initiated sounds in an auditory stream: An ERP study. Psychophysiology 48, 1276-1283. https://doi.org/10.1111/j.1469-8986.2011.01196.x

Balleine, B.W., Delgado, M.R., Hikosaka, O., 2007. The role of the dorsal striatum in reward and decision-making. J. Neurosci. 27, 8161-8165. https://doi.org/10.1523/JNEUROSCI.1554-07.2007

Bansal, S., Ford, J.M., Spering, M., 2018. The function and failure of sensory predictions. Ann. N. Y. Acad. Sci. 1426, 199-220. https://doi.org/10.1111/nyas.13686

Bastin, J., Deman, P., David, O., Gueguen, M., Benis, D., Minotti, L., Hoffman, D., Combrisson, E., Kujala, J., Perrone-Bertolotti, M., Kahane, P., Lachaux, J.P., Jerbi, K., 2017. Direct Recordings from Human Anterior Insula Reveal its Leading Role within the Error-Monitoring Network. Cereb. Cortex 27, 1545-1557. https://doi.org/10.1093/cercor/bhv352

Bates, D., Mächler, M., Bolker, B., Walker, S., 2015. Fitting Linear Mixed-Effects Models Using Ime4 | Bates | Journal of Statistical Software. J. Stat. Softw. 67.

Benazet, M., Thénault, F., Whittingstall, K., Bernier, P.M., 2016. Attenuation of visual 
668

669

670

671

672

673

674

675

676

677

678

679

680

681

682

reafferent signals in the parietal cortex during voluntary movement. J. Neurophysiol. 116, 1831-1839. https://doi.org/10.1152/jn.00231.2016

Beran, M.J., Smith, J.D., Redford, J.S., Washburn, D.A., 2006. Rhesus macaques (Macaca mulatto) monitor uncertainty during numerosity judgments. J. Exp. Psychol. Anim. Behav. Process. 32, 111-119. https://doi.org/10.1037/0097-7403.32.2.111

Bertsch, S., Pesta, B.J., Wiscott, R., McDaniel, M.A., 2007. The generation effect: A metaanalytic review. Mem. Cogn. 35, 201-210. https://doi.org/10.3758/BF03193441

Brainard, D.H., 1997. The Psychophysics Toolbox. Spat. Vis. 10, 433-436. https://doi.org/10.1163/156856897X00357

Brooks, J.X., Cullen, K.E., 2019. Predictive Sensing: The Role of Motor Signals in Sensory Processing. Biol. Psychiatry Cogn. Neurosci. Neuroimaging 4, 842-850. https://doi.org/10.1016/j.bpsc.2019.06.003

Brown, H., Adams, R.A., Parees, I., Edwards, M., Friston, K., 2013. Active inference, sensory attenuation and illusions. Cogn. Process. 14, 411-427. https://doi.org/10.1007/s10339013-0571-3

Bürkner, P.C., 2017. brms: An R package for Bayesian multilevel models using Stan. J. Stat. Softw. https://doi.org/10.18637/jss.v080.i01

Burr, D.C., Anobile, G., Arrighi, R., 2018. Psychophysical evidence for the number sense. Philos. Trans. R. Soc. B Biol. Sci. 373. https://doi.org/10.1098/rstb.2017.0045

Cappelletti, M., Barth, H., Fregni, F., Spelke, E.S., Pascual-Leone, A., 2007. rTMS over the intraparietal sulcus disrupts numerosity processing. Exp. Brain Res. 179, 631-642. 
https://doi.org/10.1007/s00221-006-0820-0

Castronovo, J., Seron, X., 2007. Numerical Estimation in Blind Subjects: Evidence of the Impact of Blindness and Its Following Experience. J. Exp. Psychol. Hum. Percept. Perform. 33, 1089-1106. https://doi.org/10.1037/0096-1523.33.5.1089

Cohen, L., Dehaene, S., 1996. Cerebral Networks for Number Processing: Evdence from a Case of Posterior Callosal Lesion. Neurocase 2, 155-174. https://doi.org/10.1080/13554799608402394

Cowan, N., 2010. The magical mystery four: How is working memory capacity limited, and why? Curr. Dir. Psychol. Sci. 19, 51-57. https://doi.org/10.1177/0963721409359277

Cromwell, H.C., Mears, R.P., Wan, L., Boutros, N.N., 2008. Sensory gating: A translational effort from basic to clinical science. Clin. EEG Neurosci. 39, 69-72. https://doi.org/10.1177/155005940803900209

Dehaene, S., 1997. The Number Sense. Oxford Univ. Press.

DeWitt, I., Rauschecker, J.P., 2012. Phoneme and word recognition in the auditory ventral stream. Proc. Natl. Acad. Sci. U. S. A. 109, 505-514. https://doi.org/10.1073/pnas.1113427109

Dormal, V., Andres, M., Pesenti, M., 2012. Contribution of the right intraparietal sulcus to numerosity and length processing: An fMRI-guided TMS study. Cortex 48, 623-629. https://doi.org/10.1016/j.cortex.2011.05.019

Duyan, Y.A., Balcı, F., 2019. Metric error monitoring in the numerical estimates. Conscious. Cogn. https://doi.org/10.1016/j.concog.2018.11.011 
Duyan, Y.A., Balcl, F., 2018. Numerical error monitoring. Psychon. Bull. Rev. https://doi.org/10.3758/s13423-018-1506-x

Farrer, C., Frith, C.D., 2002. Experiencing oneself vs another person as being the cause of an action: The neural correlates of the experience of agency. Neuroimage 15, 596-603. https://doi.org/10.1006/nimg.2001.1009

Ferrand, L., Alario, F.-X., 1998. Normes d'associations verbales pour 366 noms d'objets concrets. Annee. Psychol. 98, 659-709. https://doi.org/10.3406/psy.1998.28564

Fleming, S.M., Lau, H.C., 2014. How to measure metacognition. Front. Hum. Neurosci. 8, 19. https://doi.org/10.3389/fnhum.2014.00443

Friston, K.J., Buechel, C., Fink, G.R., Morris, J., Rolls, E., Dolan, R.J., 1997. Psychophysiological and modulatory interactions in neuroimaging. Neuroimage 6, 218-229. https://doi.org/10.1006/nimg.1997.0291

Hickok, G., 2012. Computational neuroanatomy of speech production. Nat. Rev. Neurosci. 13, 135-145. https://doi.org/10.1038/nrn3158

Hughes, G., Waszak, F., 2011. ERP correlates of action effect prediction and visual sensory attenuation in voluntary action. Neuroimage 56, 1632-1640. https://doi.org/10.1016/j.neuroimage.2011.02.057

Jack, B.N., Le Pelley, M.E., Han, N., Harris, A.W.F., Spencer, K.M., Whitford, T.J., 2019. Inner speech is accompanied by a temporally-precise and content-specific corollary discharge. Neuroimage

198, 170-180. https://doi.org/10.1016/j.neuroimage.2019.04.038 
Kansaku, K., Carver, B., Johnson, A., Matsuda, K., Sadato, N., Hallett, M., 2007. The role of the human ventral premotor cortex in counting successive stimuli. Exp. Brain Res. 178, 339-350. https://doi.org/10.1007/s00221-006-0736-8

Kilteni, K., Andersson, B.J., Houborg, C., Ehrsson, H.H., 2018. Motor imagery involves predicting the sensory consequences of the imagined movement. Nat. Commun. 9, 19. https://doi.org/10.1038/s41467-018-03989-0

Kilteni, K., Ehrsson, H., 2020. Functional connectivity between the cerebellum and somatosensory areas implements the attenuation of self-generated touch. J. Neurosci. 40, 894-906. https://doi.org/10.1523/JNEUROSCI.1732-19.2019

Kim, B.S., Im, H.I., 2019. The role of the dorsal striatum in choice impulsivity. Ann. N. Y. Acad. Sci. 1451, 92-111. https://doi.org/10.1111/nyas.13961

Kircher, T., David, A., 2003. The Self in Neuroscience and Psychiatry, The self in neuroscience and psychiatry. Cambridge University Press, Cambridge. https://doi.org/10.1017/CBO9780511543708

Kleiner, M., Brainard, D.H., Pelli, D.G., Broussard, C., Wolf, T., Niehorster, D., 2007. What's new in Psychtoolbox-3? Perception 36, S14. https://doi.org/10.1068/v070821

Kok, P., Jehee, J.F.M., de Lange, F.P., 2012. Less Is More: Expectation Sharpens Representations in the Primary Visual Cortex. Neuron 75, 265-270. https://doi.org/10.1016/j.neuron.2012.04.034

Kuznetsova, A., Brockhoff, P.B., Christensen, R.H.B., 2017. ImerTest Package: Tests in Linear Mixed Effects Models . J. Stat. Softw. 82. https://doi.org/10.18637/jss.v082.i13 
Legrand, D., 2006. The bodily self: The sensori-motor roots of pre-reflective selfconsciousness. Phenomenol. Cogn. Sci. 5, 89-118. https://doi.org/10.1007/s11097005-9015-6

Lezak, M.D., 1995. Neuropsychological assessment (3rd ed.), Neuropsychological assessment (3rd ed.).

Liebenthal, E., Binder, J.R., Spitzer, S.M., Possing, E.T., Medler, D.A., 2005. Neural substrates of phonemic perception. Cereb. Cortex 15, 1621-1631. https://doi.org/10.1093/cercor/bhi040

Lima, C.F., Krishnan, S., Scott, S.K., 2016. Roles of Supplementary Motor Areas in Auditory Processing and Auditory Imagery. Trends Neurosci. 39, 527-542. https://doi.org/10.1016/j.tins.2016.06.003

Luke, S.G., 2017. Evaluating significance in linear mixed-effects models in R. Behav. Res. Methods 49, 1494-1502. https://doi.org/10.3758/s13428-016-0809-y

McLaren, D.G., Ries, M.L., Xu, G., Johnson, S.C., 2012. A generalized form of contextdependent psychophysiological interactions (gPPI): A comparison to standard $\begin{array}{llll}\text { approaches. } & \text { Neuroimage } & \text { 1277-1286. }\end{array}$ https://doi.org/10.1016/j.neuroimage.2012.03.068

Miall, R.C., Wolpert, D.M., 1996. Forward models for physiological motor control. Neural Networks. https://doi.org/10.1016/S0893-6080(96)00035-4

Oldfield, R.C., 1971. The assessment and analysis of handedness: The Edinburgh inventory. Neuropsychologia 9, 97-113. https://doi.org/10.1016/0028-3932(71)90067-4 
Pelli, D.G., 1997. The VideoToolbox software for visual psychophysics: Transforming $\begin{array}{lllll}\text { numbers into } & \text { movies. } & \text { Spat. } & \text { Vis. }\end{array}$ https://doi.org/10.1163/156856897X00366

Pereira, M., Faivre, N., Iturrate, I., Wirthlin, M., Serafini, L., Martin, S., Desvachez, A., Blanke, O., Van De Ville, D., Millán, J. del R., 2020. Disentangling the origins of confidence in speeded perceptual judgments through multimodal imaging. Proc. Natl. Acad. Sci. 117, 8382-8390. https://doi.org/10.1073/pnas.1918335117

Piazza, M., Izard, V., Pinel, P., Le Bihan, D., Dehaene, S., 2004. Tuning curves for approximate numerosity in the human intraparietal sulcus. Neuron 44, 547-555. https://doi.org/10.1016/j.neuron.2004.10.014

Piazza, M., Mechelli, A., Price, C.J., Butterworth, B., 2006. Exact and approximate judgements of visual and auditory numerosity: An fMRI study. Brain Res. 1106, 177188. https://doi.org/10.1016/j.brainres.2006.05.104

Power, J.D., Barnes, K.A., Snyder, A.Z., Schlaggar, B.L., Petersen, S.E., 2012. Spurious but systematic correlations in functional connectivity MRI networks arise from subject $\begin{array}{lll}\text { motion. } & \text { Neuroimage } & \text { 2142-2154. }\end{array}$ https://doi.org/10.1016/j.neuroimage.2011.10.018

Press, C., Kok, P., Yon, D., 2020. The Perceptual Prediction Paradox. Trends Cogn. Sci. 24, 1324. https://doi.org/10.1016/j.tics.2019.11.003

Reinert, R.M., Hartmann, M., Huber, S., Moeller, K., 2019. Unbounded number line estimation as a measure of numerical estimation. PLoS One 14, 1-16. https://doi.org/10.1371/journal.pone.0213102 
Rummell, B.P., Klee, J.L., Sigurdsson, T., 2016. Attenuation of responses to self-generated sounds in auditory cortical neurons. J. Neurosci. 36, 12010-12026. https://doi.org/10.1523/JNEUROSCI.1564-16.2016

Scott, M., Yeung, H.H., Gick, B., Werker, J.F., 2013. Inner speech captures the perception of external speech. J. Acoust. Soc. Am. 133, EL286-EL292. https://doi.org/10.1121/1.4794932

Serino, A., Pozeg, P., Bernasconi, F., Solcà, M., Hara, M., Progin, P., Stripeikyte, G., Dhanis, H., Salomon, R., Bleuler, H., Rognini, G., Blanke, O., 2021. Thought consciousness and source monitoring depend on robotically controlled sensorimotor conflicts and illusory states. iScience 24, 101955. https://doi.org/10.1016/j.isci.2020.101955

Shergill, S.S., White, T.P., Joyce, D.W., Bays, P.M., Wolpert, D.M., Frith, C.D., 2013. Modulation of somatosensory processing by action. Neuroimage 70, 356-362. https://doi.org/10.1016/j.neuroimage.2012.12.043

Slamecka, N.J., Graf, P., 1978. The generation effect: Delineation of a phenomenon. J. Exp. Psychol. Hum. Learn. Mem. 4, 592-604. https://doi.org/10.1037/0278-7393.4.6.592

Stripeikyte, G., Pereira, M., Rognini, G., Potheegadoo, J., Blanke, O., \& Faivre, N. (2021). Increased functional connectivity of the intraparietal sulcus underlies the attenuation of numerosity estimations for self-generated words [Data set]. Zenodo. http://doi.org/10.5281/zenodo.4925909

Thomas, E.R., Yon, D., Lange, F.P. de, Press, C., 2020. Action enhances predicted touch. bioRxiv 2020.03.26.007559. https://doi.org/10.1101/2020.03.26.007559

Tian, X., Poeppel, D., 2010. Mental imagery of speech and movement implicates the 
dynamics of internal forward models. Front. Psychol. 1, 1-23. https://doi.org/10.3389/fpsyg.2010.00166

Tian, X., Zarate, J.M., Poeppel, D., 2016. Mental imagery of speech implicates two mechanisms of perceptual reactivation. Cortex 77, 1-12. https://doi.org/10.1016/j.cortex.2016.01.002

Timm, J., SanMiguel, I., Keil, J., Schröger, E., Schönwiesner, M., 2014. Motor Intention Determines Sensory Attenuation of Brain Responses to Self-initiated Sounds. J. Cogn. Neurosci. 26, 1481-1489. https://doi.org/10.1162/jocn_a_00552

Tzourio-Mazoyer, N., Landeau, B., Papathanassiou, D., Crivello, F., Etard, O., Delcroix, N., Mazoyer, B., Joliot, M., 2002. Automated anatomical labeling of activations in SPM using a macroscopic anatomical parcellation of the MNI MRI single-subject brain. Neuroimage 15, 273-289. https://doi.org/10.1006/nimg.2001.0978

Ullsperger, M., Harsay, H.A., Wessel, J.R., Ridderinkhof, K.R., 2010. Conscious perception of errors and its relation to the anterior insula. Brain Struct. Funct. 214, 629-643. https://doi.org/10.1007/s00429-010-0261-1

Vaccaro, A.G., Fleming, S.M., 2018. Thinking about thinking: A coordinate-based metaanalysis of neuroimaging studies of metacognitive judgements. Brain Neurosci. Adv. 2, 239821281881059. https://doi.org/10.1177/2398212818810591

van de Ven, V., Esposito, F., Christoffels, I.K., 2009. Neural network of speech monitoring overlaps with overt speech production and comprehension networks: A sequential spatial and temporal ICA study. Neuroimage 47, 1982-1991. https://doi.org/10.1016/j.neuroimage.2009.05.057 
Whitford, T.J., 2019. Speaking-Induced Suppression of the Auditory Cortex in Humans and Its Relevance to Schizophrenia. Biol. Psychiatry Cogn. Neurosci. Neuroimaging 4, 791804. https://doi.org/10.1016/j.bpsc.2019.05.011

Whitford, T.J., Jack, B.N., Pearson, D., Griffiths, O., Luque, D., Harris, A.W.F., Spencer, K.M., Le Pelley, M.E., 2017. Neurophysiological evidence of efference copies to inner speech. Elife 6, 1-23. https://doi.org/10.7554/eLife.28197

Wolpert, D.M., Flanagan, J.R., 2001. Motor prediction. Curr. Biol. 11, 729-732. https://doi.org/10.1016/s0960-9822(01)00432-8

847

848 
Figure 1. Schematic representation of the general task flow: active (top) and passive (bottom) conditions consisting of instruction, word generation/listening, numerosity and error estimation. Each condition was repeated 4 times per block. Auditory and visual instructions were provided in French. Simulated distribution of derived behavioral performance measures using numerosity and error estimation values are depicted in Figure $1-1$.

Figure 2. Behavioral results. (A) Individual numerosity performance in the active (red) and passive (blue) conditions. A value of zero represents ideal performance, where the reported and actual number of words match. Each dot represents a participant mean numerosity performance. Grey lines connect performance from the same participant across conditions.

(B) Mixed-effects linear regression between numerosity estimation (reported number of words) and an actual number of words. Reference dashed line with a slope equal to 1 represents ideal performance. Thick lines represent the global model fit, while thin lines depict individual estimates. Individual plots are depicted in Figure 2-1. (C) Mean slope coefficient estimates with $2.5 \%$ and $97.5 \%$ confidence intervals.

Figure 3. Percentage of simulated accurate numerosity estimations (occurrences of value 10) in normal distribution centred on 10 with varying standard deviation. Simulation was iterated 10000 times. Reference dashed line of $19.5 \%$ absolute numerosity estimations accuracy (as observed in the experiment empirical data) occurs with the standard deviation 
871 of 2. Each dot represents a single iteration of the data simulation (overlapping dots are

872 depicted in higher color intensity).

873 Figure 4. Neural correlates of numerosity performance. (A) Main effect (active+passive 874 conditions) of the parametric modulation of numerosity performance during numerosity 875 estimation in the right intraparietal sulcus (IPS, depicted in green). (B) Box plot of the 876 corresponding individual beta estimates in the right IPS. Each dot represents a participant 877 mean beta estimates. Grey lines connect beta estimates from the same participant across 878 conditions. (C) Overlap between the parametric modulation of numerosity performance 879 during numerosity estimation (depicted in green) main effect (active + passive conditions) at 880 the lower statistical threshold of peak level uncorrected $p<0.005$ (exploratory analyses) and 881 parametric modulation of performance monitoring during the error estimation (active vs. passive condition) (depicted in purple); peak level uncorrected $p<0.001$.

Figure 5. PPI analysis: numerosity estimation. (A) Increased functional connectivity in the active vs. passive condition between the right intraparietal sulcus seed (IPS, depicted in green) and bilateral supplementary motor area (SMA), left inferior parietal lobule (IPL) and superior temporal gyri (STG), depicted in red. (C) Box plots of the corresponding beta estimates for each significant PPI cluster. Each dot represents a participant mean beta estimates. Grey lines connect beta estimates from the same participant across conditions.

Figure 6. Neural correlates of performance monitoring. (A) Decreased neural activity during the active vs. passive condition in the right putamen and insula bilaterally (depicted in blue). 
Increased activity in the active vs. passive condition in the left caudate nucleus (depicted in red). (B) Box plots of the corresponding beta estimates in the active (red) and passive (blue) conditions for each significant cluster observed. Each dot represents a participant mean beta estimates. Grey lines connect beta estimates from the same participant across conditions.

Figure 7. Parametric modulation of performance monitoring. (A) Positive association between the trial-wise performance monitoring and BOLD signal during performance monitoring in the active vs. passive condition in the left intraparietal sulcus (IPS, depicted in purple). (B) Box plot of corresponding beta estimates in the left IPS in the active (blue) and passive (red) conditions. Each dot represents a participant mean beta estimates. Grey lines connect beta estimates from the same participant across conditions.

\section{Tables}

Table 1. Numerosity estimation: difference between active and passive conditions. Brain areas showing a difference in activity between the active and passive condition during the numerosity estimation phase, independent of parametric modulation. The activation clusters of numerosity estimation main effect are detailed in Table 1-1. Voxel level $p<0.001$ uncorrected, cluster threshold at $\mathrm{p}<0.05$ FWE corrected. BA - Broadmann area, $\mathrm{k}$ - cluster size, $R$ - right hemisphere, $L$ - left hemisphere, $R$ - right hemisphere

Table 2. Parametric modulation of numerosity performance (exploratory analyses at lower statistical threshold). Brain areas in which the BOLD signal correlates with the trial-by-trial 
behavioral measures of numerosity performance during the numerosity estimation phase. Voxel level $p<0.005$ uncorrected, cluster threshold at $p<0.05$ FWE corrected. BA Broadmann area, $\mathrm{k}$ - cluster size, $\mathrm{R}$ - right hemisphere, $\mathrm{L}$ - left hemisphere

Table 3. PPI analysis: numerosity estimation. Brain areas identified through PPI analysis seeding from the right intraparietal sulcus during numerosity estimation. Voxel level $p<0.001$ uncorrected, cluster threshold at $p<0.05$ FWE corrected. BA - Broadmann area, $k-$ cluster size, $\mathrm{R}$ - right hemisphere, $\mathrm{L}$ - left hemisphere

Table 4. Error estimation: difference between active and passive conditions. Brain areas showing a difference in activity between the active and passive condition during the error estimation phase, independent of parametric modulation. The activation clusters of error estimation main effect are detailed in Table 4-1. Voxel level $p<0.001$ uncorrected, cluster threshold at $\mathrm{p}<0.05$ FWE corrected. BA - Broadmann area, $\mathrm{k}$ - cluster size, $\mathrm{R}$ - right hemisphere, $L$ - left hemisphere

\section{Extended data legends}

Figure 1-1. Data simulation of behavioral performance measures. Data was simulated with pseudorandom generation of an actual number of words and numerosity estimations ranging between 5 and 20. Error estimation varied between 0 and 5 as set during the task design. (A) Simulated numerosity performance. Increasing intensity of red represent linear increase of overestimation (positive values of numerosity performance), while increasing 
intensity in blue represent linear increase of underestimation (negative values of numerosity performance). (B) Simulated performance monitoring when the number of words is constant (10 or 15 words). The performance monitoring value is depicted in the heatmap changing from blue (being correct) to red (worse performance monitoring).

Figure 2-1. Mixed-effects linear regression between numerosity estimation (reported number of words) and an actual number of words depicted for each participant. Reference dashed line with a slope equal to 1 represents ideal performance. Thick lines represent the individual model fit per condition (active: red and passive: blue). Each dot represents a participant's single trial performance (overlapping dots are depicted in higher color intensity).

Table 1-1. Numerosity estimation: main effect (active + passive conditions). Brain areas activated during the numerosity estimation phase independent of parametric modulation. Voxel level $p<0.001$ uncorrected, cluster threshold at $p<0.05$ FWE corrected. BA Broadmann area, $k$ - cluster size, $R$ - right hemisphere, $L$ - left hemisphere, $R$ - right hemisphere

Table 4-1. Error estimation: main effect (active + passive conditions). Brain areas with activations during the error estimation phase, independent of parametric modulation. Voxel level $p<0.001$ uncorrected, cluster threshold at $p<0.05$ FWE corrected. BA - Broadmann area, $\mathrm{k}$ - cluster size, $\mathrm{R}$ - right hemisphere, $\mathrm{L}$ - left hemisphere, $\mathrm{R}$ - right hemisphere 

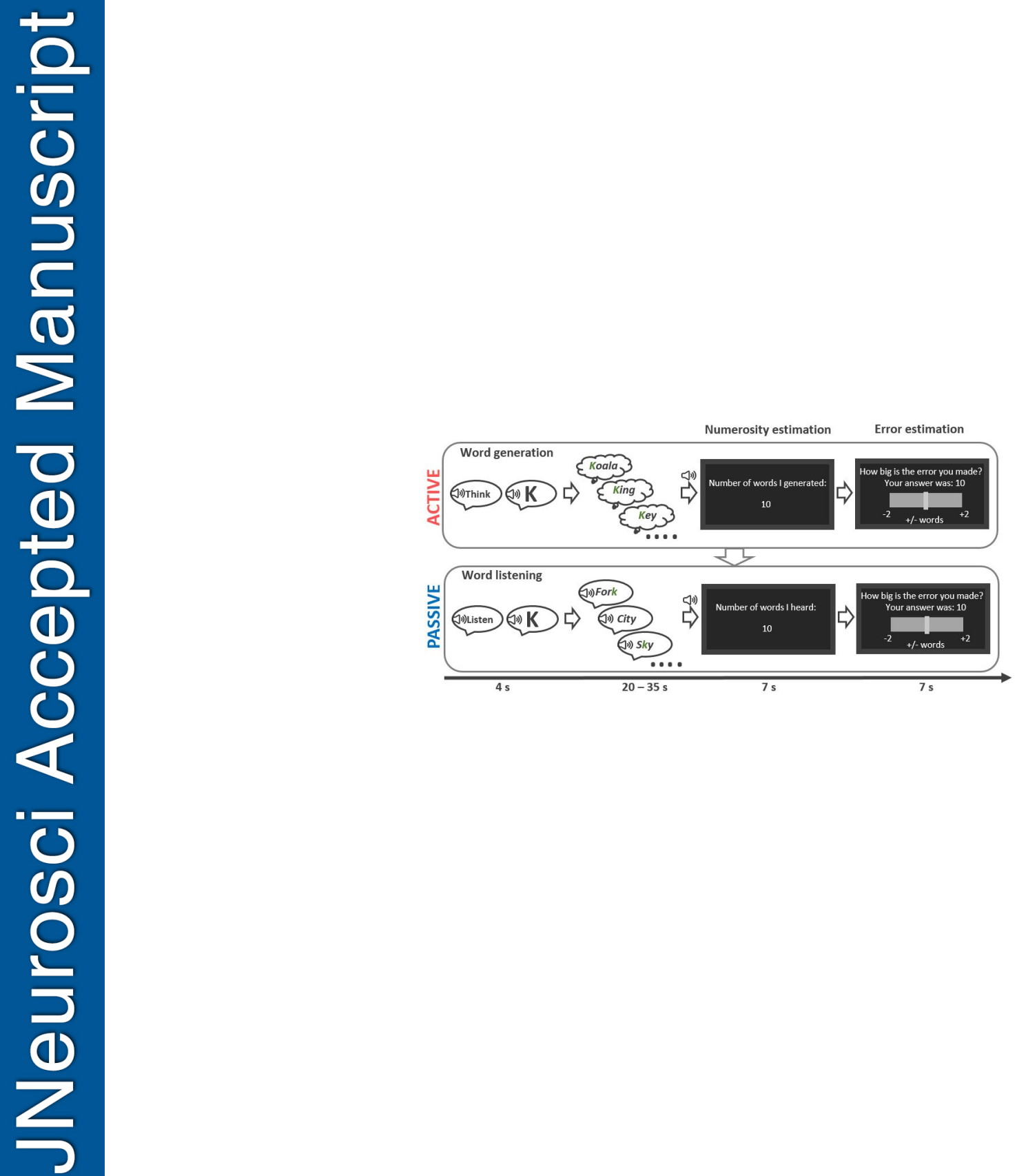


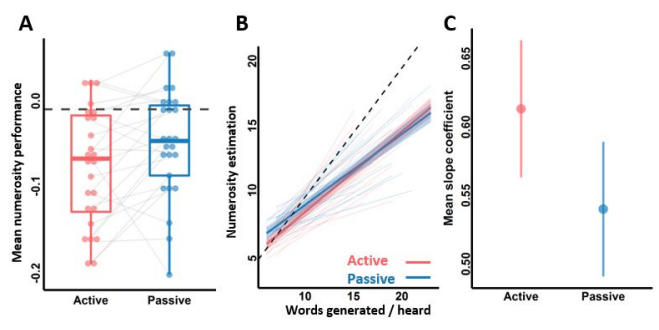




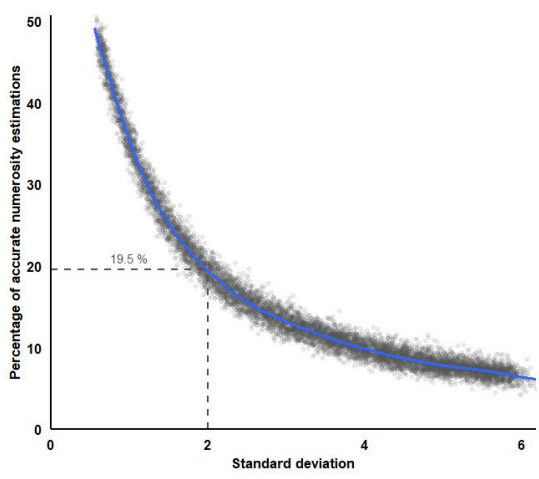




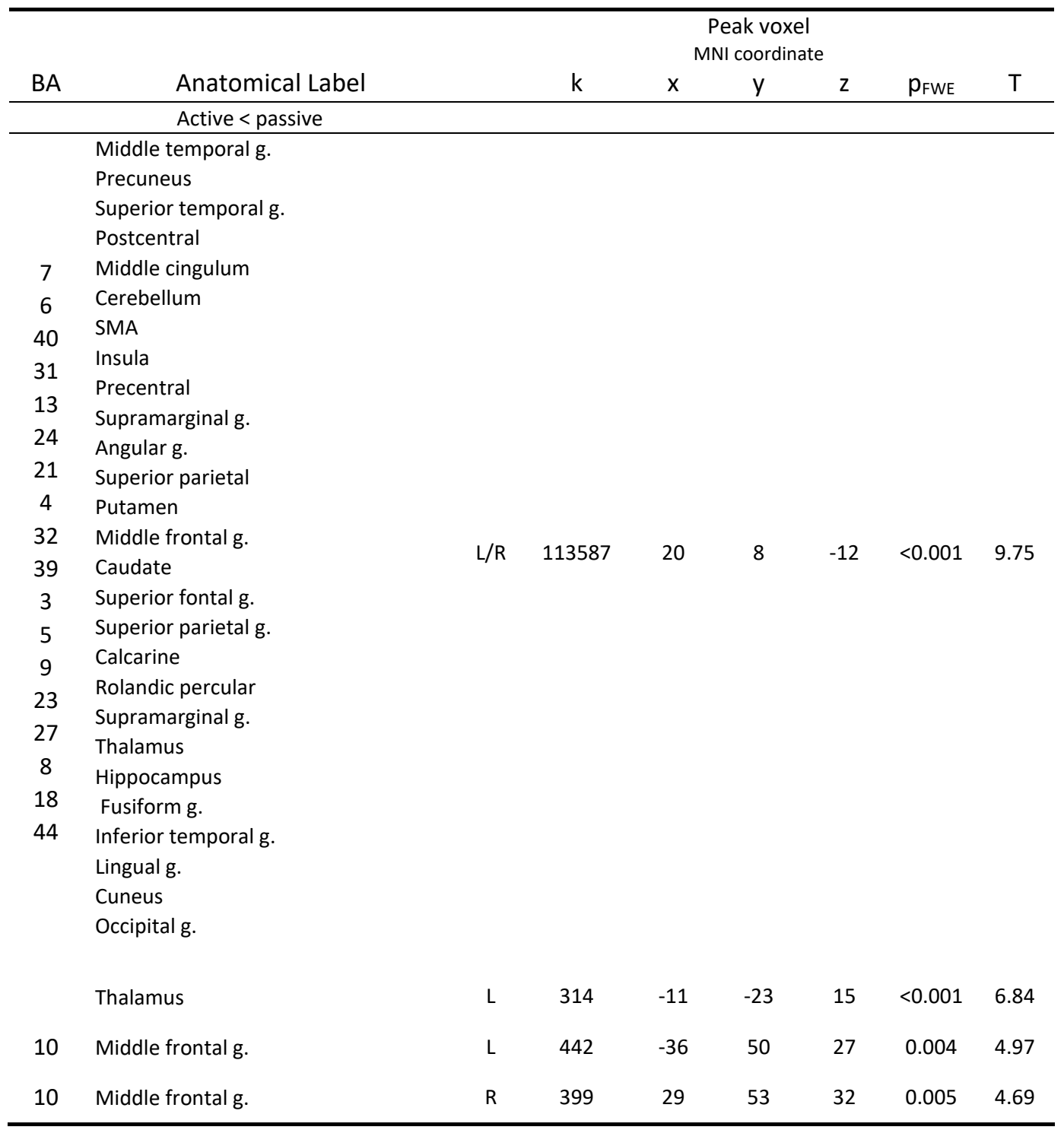

Table 1. Numerosity estimation: difference between active and passive conditions. Brain areas showing a difference in activity between the active and passive condition during the numerosity estimation phase, independent of parametric modulation. The activation clusters of numerosity estimation main effect are detailed in Table 1-1. Voxel level $p<0.001$ uncorrected, cluster threshold at $\mathrm{p}<0.05$ FWE corrected. BA - Broadmann area, $\mathrm{k}-$ cluster size, $R$ - right hemisphere, $L$ - left hemisphere, $R$ - right hemisphere 


\begin{tabular}{cccccccc}
\hline & \multicolumn{7}{c}{ Peak voxel } \\
BA & Anatomical Label & $\mathrm{k}$ & $\mathrm{x}$ & $\mathrm{y}$ & $\mathrm{z}$ & $\mathrm{p}_{\mathrm{FWE}}$ & $\mathrm{T}$ \\
\hline \multicolumn{1}{c}{ Main effect (active + passive) } \\
\hline 7 & $\mathrm{R}$ & 466 & 29 & -65 & 50 & 0.004 & 20 \\
\hline $\begin{array}{l}\text { Intraparietal sulcus } \\
\text { (superior parietal g., angular g.) } \\
\text { Intraparietal sulcus } \\
\text { (superior/inferior parietal g., } \\
\text { middle occipital g.) }\end{array}$ & $\mathrm{L}$ & 758 & -30 & -63 & 42 & 0.000 & 15.9 \\
\hline
\end{tabular}

Table 2. Parametric modulation of numerosity performance (exploratory analyses at lower statistical threshold). Brain areas in which the BOLD signal correlates with the trial-by-trial behavioral measures of numerosity performance during the numerosity estimation phase. Voxel level $p<0.005$ uncorrected, cluster threshold at $p<0.05$ FWE corrected. BA Broadmann area, $\mathrm{k}$ - cluster size, $\mathrm{R}$ - right hemisphere, $\mathrm{L}$ - left hemisphere 


\begin{tabular}{|c|c|c|c|c|c|c|c|c|}
\hline \multirow[b]{2}{*}{ BA } & \multirow[b]{2}{*}{ Anatomical Label } & \multicolumn{7}{|c|}{$\begin{array}{c}\text { Peak voxel } \\
\text { MNI coordinate }\end{array}$} \\
\hline & & & k & $x$ & $\mathrm{y}$ & z & $p_{\mathrm{FWE}}$ & $\mathrm{T}$ \\
\hline \multicolumn{9}{|c|}{ Active $>$ passive } \\
\hline $\begin{array}{l}41 \\
42\end{array}$ & Superior temporal g. & L & 177 & -54 & -20 & 6 & 0.038 & 5.14 \\
\hline 40 & $\begin{array}{l}\text { Inferior parietal lobule } \\
\text { (supramarginal g., } \\
\text { parietal inferior g.) }\end{array}$ & $\mathrm{L}$ & 291 & -44 & -42 & 29 & 0.003 & 5.14 \\
\hline 6 & Supplementary motor area & $L / R$ & 167 & -3 & -30 & 63 & 0.049 & 4.59 \\
\hline
\end{tabular}

Table 3. PPI analysis: numerosity estimation. Brain areas identified through PPI analysis seeding from the right intraparietal sulcus during numerosity estimation. Voxel level $p<0.001$ uncorrected, cluster threshold at $\mathrm{p}<0.05$ FWE corrected. BA - Broadmann area, $\mathrm{k}$ - cluster size, $\mathrm{R}$ - right hemisphere, $\mathrm{L}-$ left hemisphere 


\begin{tabular}{|c|c|c|c|c|c|c|c|c|}
\hline \multirow[b]{2}{*}{ BA } & \multirow[b]{2}{*}{ Anatomical Label } & \multicolumn{7}{|c|}{$\begin{array}{l}\text { Peak voxel } \\
\text { MNI coordinate }\end{array}$} \\
\hline & & & k & $x$ & $\mathrm{y}$ & z & $p_{F W E}$ & $\mathrm{~T}$ \\
\hline \multicolumn{9}{|c|}{ Active $>$ passive } \\
\hline & Caudate nucleus & L & 336 & -15 & 30 & -6 & 0.004 & 5.76 \\
\hline \multicolumn{9}{|c|}{ Active $<$ passive } \\
\hline $\begin{array}{l}45 \\
47 \\
13\end{array}$ & $\begin{array}{l}\text { Insula, } \\
\text { inferior frontal g. triangular part }\end{array}$ & $\mathrm{L}$ & 392 & -33 & 24 & -6 & 0.002 & 5.3 \\
\hline & Putamen & $\mathrm{R}$ & 209 & 20 & 12 & -6 & 0.043 & 5.2 \\
\hline $\begin{array}{l}13 \\
47 \\
45\end{array}$ & $\begin{array}{l}\text { Insula, } \\
\text { inferior frontal g. triangular part }\end{array}$ & $\mathrm{R}$ & 482 & 32 & 27 & 0 & 0.000 & 4.3 \\
\hline
\end{tabular}

Table 4. Error estimation: difference between active and passive conditions. Brain areas showing a difference in activity between the active and passive condition during the error estimation phase, independent of parametric modulation. The activation clusters of error estimation main effect are detailed in Table 4-1. Voxel level $p<0.001$ uncorrected, cluster threshold at $\mathrm{p}<0.05$ FWE corrected. BA - Broadmann area, $\mathrm{k}$ - cluster size, $\mathrm{R}$ - right hemisphere, $\mathrm{L}$ - left hemisphere 